\title{
Experimental and numerical validation of an enhanced cylindrical contact force model
}

\author{
C. Pereira ${ }^{1}$, A. Ramalho ${ }^{2}$ \& J. Ambrósio ${ }^{3}$ \\ ${ }^{1}$ Department of Mechanical Engineering, \\ Polytechnic Institute of Coimbra, Portugal \\ ${ }^{2}$ Department of Mechanical Engineering, \\ University of Coimbra, Portugal \\ ${ }^{3}$ Department of Mechanical Engineering, \\ Technical University of Lisbon, Portugal
}

\begin{abstract}
Many applications in contact mechanics require that the contact force is expressed in terms of a pseudo-indentation. For spherical contact and when the assumptions of the Hertz contact model are fulfilling, it provides a continuous contact model which is able and simple to use. For cylindrical contact, Johnson proposed an accurate model but the contact force is defined as an implicit function of indentation. It requires therefore a numerical iterative procedure to obtain the contact force, which represents a difficulty when implemented in a computational code for impact simulation. A new enhanced cylindrical contact model that has the simplicity of the Hertz contact model and the accuracy of the Johnson cylindrical model was recently proposed by the authors. An experimental validation of new enhanced contact model to analyze the internal contact for different clearance and load values is presented and discussed in this work. It is concluded that the experimental validation is limited to a narrower range of conformal contact conditions. Therefore, a numerical study was conducted to evaluate the suitability of the enhanced model to analyze the internal elastostatic frictionless contact between cylindrical bodies for very low clearances and different loads. Depending on clearance and load values, the values estimated from the enhanced model were compared with numerical or experimental results. The validation of the new enhanced contact force model has been done therefore applying a hybrid methodology.
\end{abstract}

Keywords: contact mechanics, internal cylindrical contact, conformal contact, contact dynamics, finite element models, experimental validation. 


\section{Introduction}

For spherical impact geometries and for non-conformal contact conditions, the contact parameters used to define the contact force are estimated by applying the Hertz contact model [1, 2]. However, for cylindrical geometries, that can be found in common examples of mechanical engineering practice [3-6], the physical meaning of contact parameters is not so straightforward and their values are not so easily obtained [6]. Several analytical cylindrical contact force models to evaluate the relation between the indentation of the surfaces and the contact force exerted between them can be found in the literature [1,8-11]. However, since these models are based on Hertz pressure distribution, they are restricted to a limited range of conditions, in particular for conformal contact conditions where low clearances and high loads values occur simultaneously [4]. Moreover, they are iterative models, i.e., since the contact force is defined as a implicit function of indentation, the use of less efficient numerical procedures is required to calculate the contact force $[2,4-7]$.

To overcome these drawbacks a new enhanced analytical cylindrical model without domain validity problems and defining the contact force as an explicit function of indentation was recently presented by the authors [12]. This model represents a good alternative for modelling the contact between cylindrical geometries, in particular for implementation in a computational code for impact analysis, avoiding inefficient iterative procedures. Nevertheless, it was derived from the approach proposed by Johnson [1]. Therefore, it is necessary to evaluate the performance of the new enhanced contact model, in particular for situations of cylindrical conformal contact. With this purpose, an experimental setup was developed which was capable of assessing the contact models proposed in analyzing the internal cylindrical conformal contact for different clearance values. Unfortunately, the experimental validation is limited to a narrower range of values. In fact, experimental clearance values as low as those verified in most of the mechanical applications allow to very high contact stiffness values which require special equipment for measuring the deformation. Since they are versatile enough to explore a wider range of variables $[13,14]$, particularly in situations of conformal contact, finite element models are an attractive alternative. Most of the works published in the literature using different finite element codes focus on the analysis and modelling of interfacial phenomena in the scale of asperity contacts, i.e., they study contact phenomena at the micro level in order to understand and foresee the variations in the interfacial material layers and wear phenomenon [15-21]. However, in the present case, conformal contact should be studied as a macro-phenomenon, because the current study is focused in global average values of contact forces. There are few studies dealing with both contacting bodies with cylindrical geometries. To analyze the internal elastostatic frictionless contact between cylindrical bodies in conditions of conformal contact a numerical study using the commercial code MARC ${ }^{\circledR}$ [22] has therefore been conducted and the results compared with those achieved experimentally and analytically. 


\section{Analytical models for cylindrical contacting bodies}

The expressions that define most of the models available in the literature describing the contact involving cylindrical geometries include logarithmic functions [1, 8-11]. This means that each cylindrical contact model has a specific validity domain of application, which depends on the clearance value and the material properties. A comparative assessment of most of these models was recently presented by the authors [2]. From this study it was concluded that the cylindrical contact model presented by Johnson [1] is the one that best describes the contact between internal contacting cylinders, when compared with other cylindrical models. Therefore, it is chosen to validate the new enhanced model. In the model suggested by Johnson, the total indentation, $\delta$, of two deformable contacting cylinders of radius $R_{i}$ and $R_{j}$ made with materials with similar elastic modulus and Poisson coefficients denoted by $E$ and $v$, respectively, and submitted to the action of a compressive load, $F_{c}$, is given by eqn. (1).

$$
f_{c}=\pi L E^{*} \frac{1}{\left\{\ln \left(\frac{4 \pi L E^{*} \Delta \mathrm{R}}{f_{c}}\right)-1\right\}} \delta
$$

In eqn. (1) the compressive load $f_{c}$ is expressed per unit of the axial length of the cylinder, $E^{*}=E / 2\left(1-v^{2}\right)$ is the composite modulus, assuming materials with similar elastic modulus and Poisson ratios denoted by $E$ and $v$, respectively, and $\Delta R$ represents the difference between cylinders' radii, $\left(R_{i}-R_{j}\right)$, corresponding to the radial clearance between the two cylindrical bodies.

For each given indentation, eqn. (1) has to be solved iteratively to evaluate the contact force that fulfils it. When used in the framework of forward dynamic analysis, this procedure is not only computationally costly but also represents a numerical difficulty for the performance of a computational code especially if a greater number of contacting bodies are involved [2, 3, 5-7]. To overcome the drawbacks associated with Johnson cylindrical contact force an alternative model, without domain validity problems, and defining the contact force as an explicit function of indentation has been recently proposed by the authors. In this model the contact force is evaluated as described by eqn. (2) [12].

$$
f_{c}=\frac{(a \Delta R+b) L E^{*}}{\Delta R} \delta^{n}
$$

In eqn (2) $a=0.49, \quad b=0.10, \quad n=Y \Delta R^{-0.005} \quad$ and $\quad \Delta R=R_{i}-R_{j}$, being $Y=1.56[\ln (1000 \Delta R)]^{-0.192}$ if $\Delta R=[0.005, \quad 0.750[$ or $\mathrm{Y}=0.0028 \Delta R+1.083$ if $\Delta R=[0.750,10.0[\mathrm{~mm}$. The remaining quantities in eqn (2) have the same meaning described for eqn (1). To discuss the suitability of the new enhanced contact model to describe the cylindrical conformal contact, an experimental setup is developed. The experimental procedure and the results obtained are presented and discussed next. 


\section{Experimental procedure}

The experimental setup, shown in fig. 1, is developed to evaluate the performance of the Johnson contact and the new enhanced models to describe conformal contact for different clearance values. The internal contact is analyzed using a cylinder, with an axial length of $60 \mathrm{~mm}$, inside an external hole with cylindrical geometry. The internal cylinder is made in DIN 34CrNiMo 6 and the external in DIN 40CrMnNiMo 8-6-4 whose yield strengths are 610 and $950 \mathrm{MPa}$, respectively. To study the effect of different clearance values, a range of internal cylinder radii is analyzed keeping constant the radius of the hole. The external cylinder is machined from a solid cubic block. After grinding of the cylinder geometry to give a radius of $30 \mathrm{~mm}$, the block is cut into two symmetrical halves. Alignment is guaranteed by two pairs of linear guides and two pairs of neck bushes. This allows the assembly to be dismantled easily, which is crucial if a range of internal cylinder radii, $R_{j}$, is to be tested by successive grinding of the internal cylinder. Thus, for each clearance value under evaluation, the internal cylinder is removed from the block, grinded to the desired radius and refitted into the block. The global set, made of internal and external cylinders, is submitted to increasing compression loads and the deformation is measured by the strain gauge extensometer of the tensile equipment of the INSTRON machine. This procedure is repeated for every clearance value under study. Radial clearance values in the range of $250 \mu \mathrm{m}$ to $9.000 \mathrm{~mm}$ are analyzed for a constant external cylinder radius, $R_{i}$, of $30 \mathrm{~mm}$.

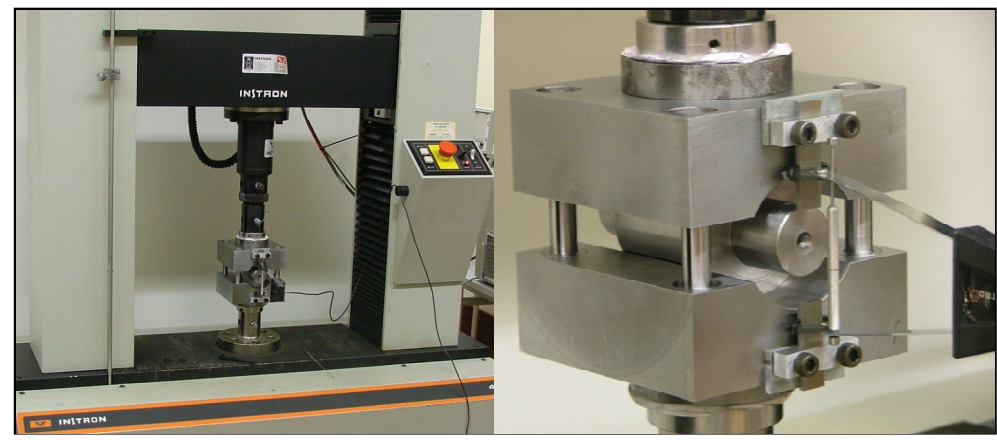

Figure 1: Experimental setup to evaluate the internal cylindrical contact.

\subsection{Experimental results vs. analytical results}

Fig. 2 illustrates the experimental and the analytical results obtained from the new enhanced and the Johnson contact models for each clearance value studied. Fig. 2a) through 2c), show that the Johnson model presents a better agreement with the experimental results than the new enhanced contact model. This behavior is not surprising and can be explained by the fact that the new model is 
optimized for a load validity domain between $1 \mathrm{~N} / \mathrm{mm}$ and $1000 \mathrm{~N} / \mathrm{mm}$. For a clearance of $0.250 \mathrm{~mm}$, if the two higher loads are neglected, differences lower than $8 \%$ can be observed between the Johnson model and the experimental results as described in fig. 2a). Fig. 2b) shows that for the clearance of $0.665 \mathrm{~mm}$, differences lower than $9.25 \%$ separate the Johnson model from the experimental results, except for the higher load in test. For the first clearance the higher loads should be neglected since they show a constant indentation value for the increasing load, which is physically inconsistent. While for the clearance of $0.665 \mathrm{~mm}$ the highest load should not be accounted for because an exaggerated increase of the indentation value is verified. Two reasons can explain these behaviors: i) some plastic deformation may have occurred due to the large load applied; and/or ii) measuring errors may have arisen due to parallelism problems, as a result of a deficient finishing process. The clearance of $1.2 \mathrm{~mm}$ leads to a slight increase in the difference between the Johnson model and the experimental results, as observed in fig. 2c). A maximum difference of $15.31 \%$ is achieved for a load value of $80 \mathrm{kN}$. For the others loads differences below $10.15 \%$ are found. For a clearance of $4 \mathrm{~mm}$ a maximum error below $5 \%$ separates the experimental results from the Johnson model compared with $9.49 \%$ using the new enhanced model, as seen in fig. 2d). Fig. 2e), which refers to clearance of $4.505 \mathrm{~mm}$, shows error values lower than $10.06 \%$ between the experimental results and the Johnson model, except for the highest load with a error value of $14.52 \%$. Concerning the new enhanced contact model, error values lower than $15.89 \%$ are achieved for all indentation values, except for the last two, in which a small increase, up to $19.14 \%$ and $23.65 \%$, respectively, can be observed. The maximum error values for a clearance value of $5.998 \mathrm{~mm}$, described in fig. 2f), are lower than $12.78 \%$ and $12.45 \%$ for the comparison between the experimental, the Johnson and the new enhanced contact models, respectively, for the whole range of load values tested. The exceptions are for the $55 \mathrm{kN}$ and $60 \mathrm{kN}$ loads, where an error of $18.62 \%$ and $16.22 \%$ are calculated between the experimental results and those from the new enhanced contact model. Fig. $2 \mathrm{~g}$ ) illustrates, for a clearance of $7 \mathrm{~mm}$, the good agreement achieved between both analytical and the experimental results where errors lower than 5\% are observed. Concerning the new enhanced contact model a small increase in the error value is verified. However, for moderate loads, load values under $55 \mathrm{kN}$, a divergence lower than $16 \%$ is obtained between the experimental results and the new enhanced contact model. For the highest clearance, represented by fig. $2 \mathrm{~h}$ ), maximum differences of $2.44 \%$ are verified between the Johnson and the new enhanced contact models. Concerning to the comparison between the analytical models and the experimental results obtained differences lower than $11,61 \%$ and $10.75 \%$ separate the Johnson and the new enhanced contact models, respectively from the experimental results.

Based on the global set of experimental results, it can be concluded that divergences of less than 15\% separate the Johnson model from the experimental results. The new enhanced contact model presents a similar agreement, but is conditioned by the load value applied. In fact, the new model deviates from the behavior exhibited by the Johnson model, particularly for high load values and low 
54 Surface Effects and Contact Mechanics X

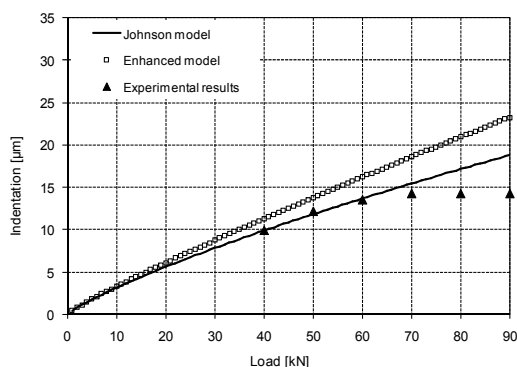

a)

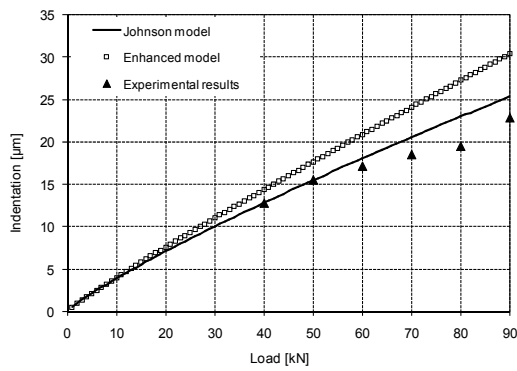

c)

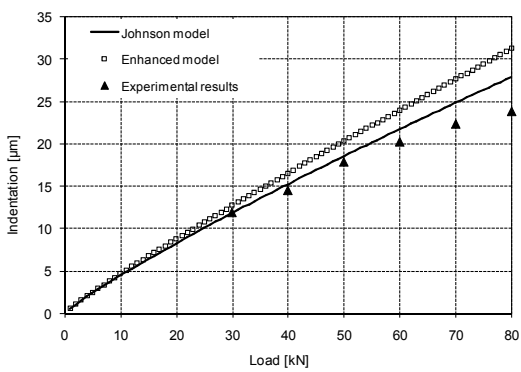

e)

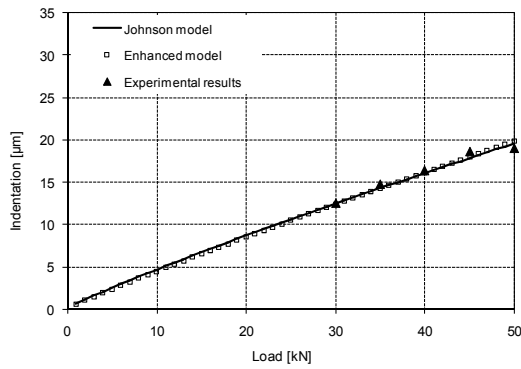

g)

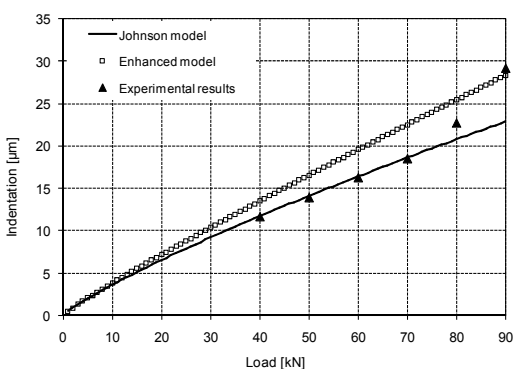

b)

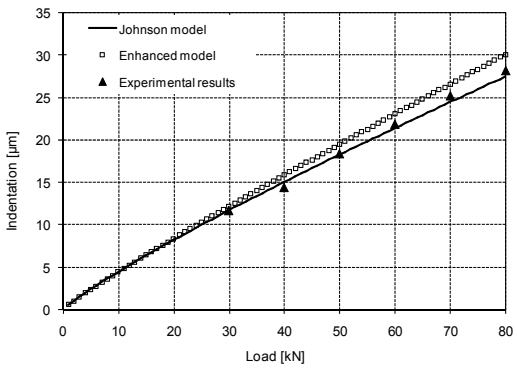

d)

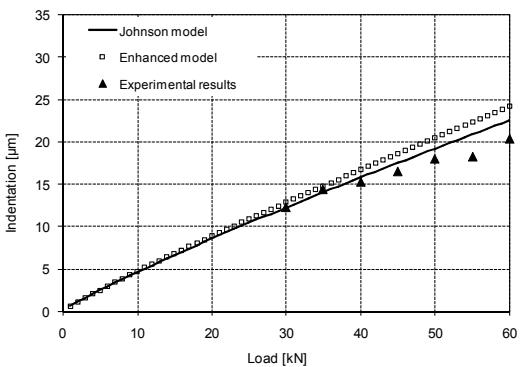

f)

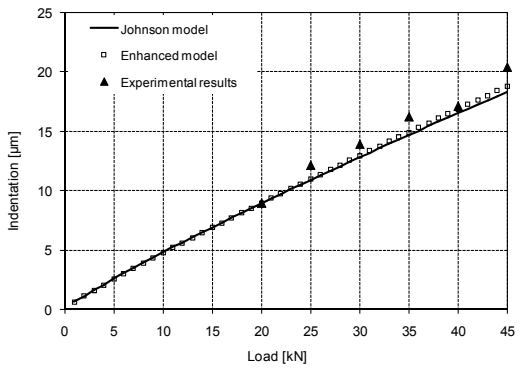

h)

Figure 2: Experimental results compared with the Johnson and the new enhanced contact models for a clearance value of: a) $0.250 \mathrm{~mm}$; b) $0.665 \mathrm{~mm}$; c) $1.200 \mathrm{~mm}$; d) $4.000 \mathrm{~mm}$; e) $4.505 \mathrm{~mm}$; f) $5.998 \mathrm{~mm}$; g) $7.000 \mathrm{~mm}$ and h) $9.000 \mathrm{~mm}$. 
clearances. This deviation seems to decrease with increasing clearance values although this behavior does not depend on the clearance value, but is only due to the range of loads applied. A good agreement was nonetheless achieved by both the Johnson model and the new model for this kind of geometries of up to load values of $30 \mathrm{kN}$, but the upper boundary of this validity domain tends to increase with increasing clearance values. Therefore, concerning the normal load, the new model seems to have a wider validity domain than the one originally identified.

Unfortunately with the machining and measuring equipment used in this research it was not achievable to test experimentally clearance values below $0.250 \mathrm{~mm}$. To achieve such low clearance values, special equipment which is unavailable to this research, is required for both machining and measuring the deformation. Therefore, to evaluate clearance values very different in dimensional terms from those analyzed experimentally a numerical study has been conducted using the nonlinear finite element code $\mathrm{MARC}^{\circledR}$, in which the direct constraint method is used to describe the contact. The comparison between numerical results and those achieved experimentally as well as with the results obtained using the new enhanced and the Johnson contact models are presented and discussed next.

\section{Validation of the new enhanced model by FEM}

Although the enhanced cylindrical model has been derived to cover a wider application range, the main goal of the authors is to apply the enhanced model in the study of the dynamics of roller chain drive mechanisms. Therefore, the dimensional values herein presented correspond to the pin/bushing contact pair as a part of a roller chain drive. In the different geometries considered in this study, the radial clearance value is obtained from the difference between an external cylinder radius of $2.245 \mathrm{~mm}$, which remains constant, and several internal cylinder radii, that corresponding to clearance values in the range of $5 \mu \mathrm{m}$ to $1.5 \mathrm{~mm}$. The mesh geometry modeling using $\mathrm{MARC}^{\circledR}$ software, for a clearance value of $0.1 \mathrm{~mm}$, is shown in fig. 3 .
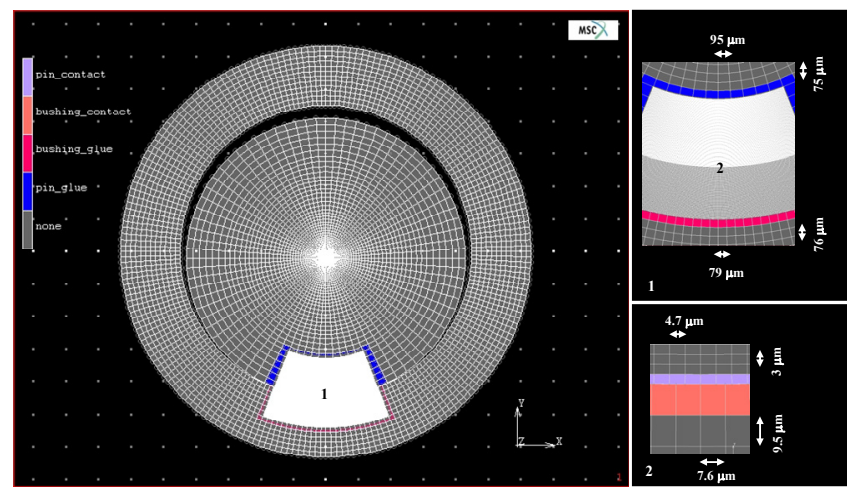

Figure 3: Deformable/deformable contact geometry modeling using MARC ${ }^{\circledR}$. 
A very fine mesh is required for good accuracy, particularly in the contact area illustrated by area 1 in fig. 3, being the mesh of remaining part of the bushing coarser. These areas are defined only by their boundary elements to reduce the computational costs. The total number of four-node plane stress isoparametric elements and nodes are 77028 and 78015, respectively. The selected mesh resulted from a meshing sensitivity study previously conducted to guarantee the accuracy of the numerical modeling. The convergence of values is achieved for a range of element sizes between 6.63 and $1.31 \mu \mathrm{m}$. The cylindrical internal contact is modeled as a deformable/deformable contact, assuming that both cylinders have equal elastic properties, with a Young modulus of $2.07 \times 10^{11} \mathrm{~Pa}$ and a Poisson ratio of 0.3 . Concerning boundary conditions, the load is applied at the center node of the horizontal line of the internal cylinder and defined as being linear and increasing over time, while displacement and rotation constraints are applied in the upper half its boundary.

\subsection{FE results vs. analytical results}

The indentation values, measured at the internal cylinder center, obtained from numerical modeling and those resulting from the application of both analytical contact models for loads of 20, 40 and $100 \mathrm{~N} / \mathrm{mm}$ are compared. Table 1 summarizes this comparison for a load value of $20 \mathrm{~N} / \mathrm{mm}$ and clearances in the range of $5 \mu \mathrm{m}$ to $1.5 \mathrm{~mm}$.

Table 1: $\quad$ Differences obtained from the comparison of $i)$ the finite element and the Johnson models; ii) the finite element and the new enhanced contact models and iii) the Johnson and the new enhanced contact models, for a load value of $20 \mathrm{~N} / \mathrm{mm}$.

\begin{tabular}{|c|c|c|c|}
\hline & FEM/Johnson & FEM/Enhanced & Johnson/Enhanced \\
\hline Clearance $(\mathrm{mm})$ & \multicolumn{3}{|c|}{ Differences $(\%)$} \\
\hline 0.005 & 9.11 & 22.59 & 17.42 \\
\hline 0.010 & 7.87 & 6.72 & 1.23 \\
\hline 0.025 & 7.00 & 0.30 & 6.71 \\
\hline 0.050 & 5.99 & 1.84 & 7.69 \\
\hline 0.100 & 4.33 & 4.00 & 0.34 \\
\hline 0.445 & 2.26 & 2.13 & 0.12 \\
\hline 0.845 & 2.30 & 3.38 & 1.11 \\
\hline 1.000 & 2.21 & 4.38 & 2.27 \\
\hline 1.500 & 2.50 & 0.63 & 3.11 \\
\hline
\end{tabular}

From table 1, it can be concluded that differences lower than $10 \%$ separate the finite element results from the results presented by the Johnson model, even for very low clearance values. However, a change of behavior is clearly observed around clearance values of $0.445 \mathrm{~mm}$. Differences lower than $3 \%$ are obtained for clearance values higher than $0.445 \mathrm{~mm}$; for low clearance values, the 
difference increase, up to $9.11 \%$, with decreasing clearance values. The same trend is observed regardless of the load value considered. A good agreement is found between the model proposed by Johnson and the new enhanced contact model. Even for extremely low clearance values a maximum difference below $8 \%$ is found between the two analytical models although a difference of $17.42 \%$ is observed for the lowest clearance value. This can be explained by the fact that the new model only uses a single expression to describe the internal contact in a large application domain. Therefore some inaccuracy can be expected, particularly for the smallest clearance values and for very low or very high loads. The comparison between finite element and analytical models is performed in terms of indentation at a point far enough from the contact, results show divergences lower than $10 \%$ and $7 \%$ in relation to the model suggested by Johnson and to the new model enhanced contact model proposed, respectively. Based on this set of results, even for extremely low clearance values, it can be concluded that a good agreement between finite element and the new enhanced and the Johnson contact models is found. In addition, differences lower than $8 \%$ separate the new enhanced contact model from the Johnson model.

\subsection{FE results vs. experimental results}

Fig. 4 establishes the comparison between the lowest clearances experimentally tested and the results obtained from the finite element and the Johnson cylindrical contact models. As it was described the new enhanced contact model was optimize for a load validity domain between $1 \mathrm{~N} / \mathrm{mm}$ and $1000 \mathrm{~N} / \mathrm{mm}$, which is substantially different from the range of normal loads applied experimentally. In fact, due to the contact conditions, magnitude of clearances and elastic contacting material properties, high normal load values are required experimentally to measure so low indentations. Therefore, this comparison is done in relation to the behavior presented by Johnson model instead to the one from new enhanced model. Differences lower than $9 \%$ separate the finite element results from those evaluated experimentally for clearances of $0.665 \mathrm{~mm}$ and $1.2 \mathrm{~mm}$, as observed in fig. 4a) and 4b), respectively. The high load values are also neglected based on the same reasons pointed out before. For the clearances of $4.505 \mathrm{~mm}$ and $5.998 \mathrm{~mm}$ a considerable increase of differences between finite element and experimental results with a maximum of $16.18 \%$ and $21.11 \%$, respectively, are obtained. For both cases, the difference is much lower as higher is the load applied and, consequently, as higher is the indentation suffered. In fact, and to avoid plastic deformation the range of loads applied experimentally decreases with increasing clearance values, which means that the contact stiffness increases and as a result small indentations can be expected. Thus, it can be concluded that the measuring equipment used is not accurate enough to measure indentation values as lower as verified for the arrangements high clearances with low loads. Probably, in these cases, the indentation values experimentally measured are of the same magnitude as the measuring equipment error. However, comparing the results provided by the finite element models and the obtained experimentally, with similar clearance values, it can be concluded that the best agreement is obtained by the finite element results, with error values below $3 \%$, while 


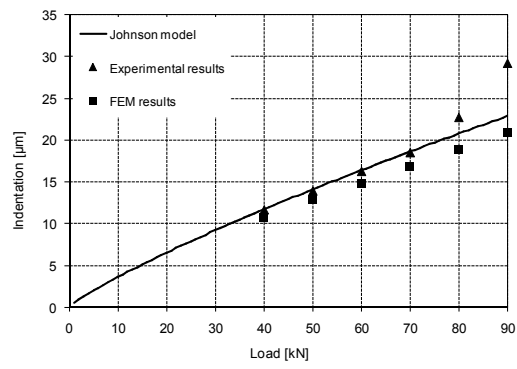

a)

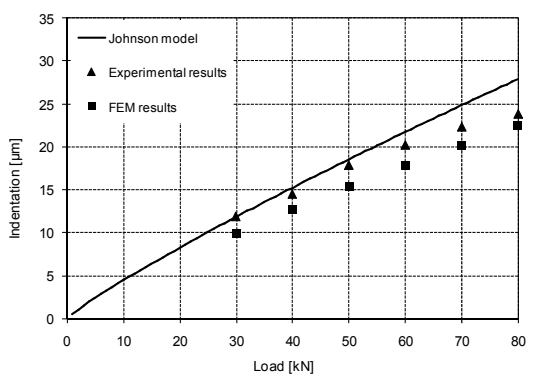

c)

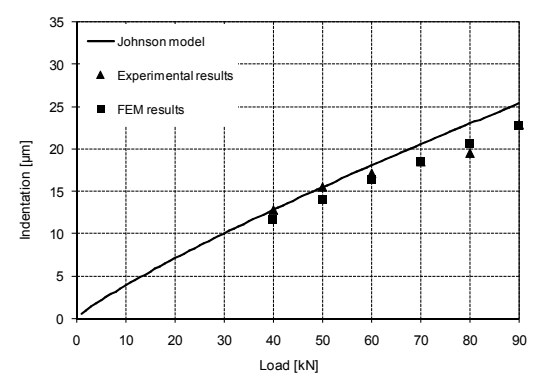

b)

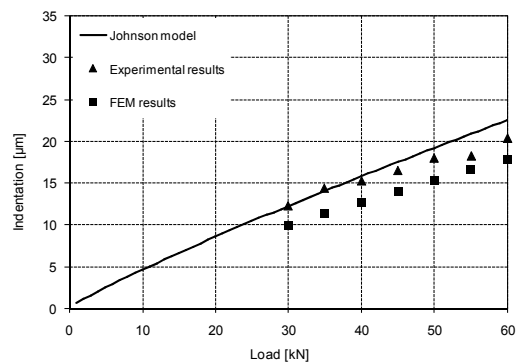

d)

Figure 4: Finite element results compared with the Johnson contact model and with experimental results for a clearance value of: a) $0.665 \mathrm{~mm}$; b) $1.200 \mathrm{~mm}$; c) $4.505 \mathrm{~mm}$ and d) $5.998 \mathrm{~mm}$.

experimental results show errors around $15 \%$. This clearly validates the numerical approach. In addition, a good agreement is obtained between the experimental values and both analytical approaches, which validates the new enhanced model by comparison to the FEM results for low clearances.

\section{Conclusions}

To validate a new enhanced cylindrical contact force model to describe the conformal contact of internal cylinders for different clearance values, an experimental setup was developed. The results reveal that the good agreement between the new enhanced contact model and the experimental results is conditioned by the normal load value applied. This behavior is not surprising because the new enhanced model was optimized for a range of normal loads lower than those required to perform the experimental tests. However, when the experimental results are compared with the analytical model proposed by Johnson a maximum divergence of less than $15 \%$ is obtained for all clearance and normal load values under analysis. The deviation between the behaviors presented by both analytical contact models seems to decrease with increasing clearance values, although this behavior does not depend directly on the clearance value but is due to the range of loads applied. A good agreement is 
nonetheless achieved by the new enhanced and the Johnson contact models, up to load values of $30 \mathrm{kN}$, but the upper boundary of this validity domain tends to increase with increasing clearance values.

Due to the limited sensitivity of the measurement displacement system used, it was not possible to analyze experimentally clearance values lower than $0.250 \mathrm{~mm}$. The experimental validation was therefore limited to a narrower range of clearances. To evaluate the performance of the new enhanced contact model, particularly for extremely low clearances, a numerical study has been conducted. A good agreement between the finite element results and those obtained the new enhanced model was found for a suitably refined mesh, even for extremely low clearance values, since maximum error values are lower than $7 \%$. In addition, it can be concluded that even for very small clearance/external radii ratios divergences lower than $8 \%$ separate the new enhanced from the Johnson contact model. Thus, based on the numerical results, it can be concluded that the new enhanced model is perfectly legitimate and suitable to describe conformal contact between cylindrical geometries.

Comparing the experimental results with the values obtained numerically for similar clearance values, the best agreement was presented by the finite element model results, with error values lower than $9 \%$. In addition, a good agreement was obtained for the experimentally tested values with both analytical approaches, which validate the new enhanced contact model by comparison with the numerical results also for low clearance values. Based on both numerical and experimental results, and for the wide range of clearance values under analysis, it can be concluded that the new enhanced contact model proposed by the authors is accurate enough to describe the conformal contact between cylindrical geometries. Furthermore, since the contact force is described as an explicit function of indentation, the new enhanced contact model is a useful alternative for modeling the contact between cylindrical geometries, especially for implementation in a computational program for dynamic impact, avoiding in this form inefficient iterative procedures.

\section{References}

[1] Johnson, K.L., Contact Mechanics, Cambridge University Press, Cambridge, 1994.

[2] Lankarani, H.M. \& Nikravesh, P.E., Continuous contact force models for impact analysis in multibody systems. Nonlinear Dynamics, 5, pp. 193-207, 1994.

[3] Pedersen, S.L., Hansen, J.M., \& Ambrósio, J.A.C., A roller chain drive model including contact with guide-bars. Multibody System Dynamics, 12(3), pp. 285-301, 2004.

[4] Pereira, M., Cândida, L. Ramalho, Amílcar \& A. Ambrósio, Jorge, A critical overview of internal and external cylinder contact force models. Nonlinear Dynamics, 63(4), pp. 681-697, 2011.

[5] Flores, P., Ambrósio, J., Pimenta Claro J. \& Lankarani, H., Kinematics and Dynamics of Multibody Systems with Imperfect Joints: Models and Case Studies, Springer, Dordrecht, The Netherlands, 2008. 
[6] Ravn, P.A., Continuous analysis method for planar multibody systems with joint clearance. Multibody System Dynamics, 2, pp. 1-24, 1998.

[7] Ravn, P., Shivaswamy, S., Alshaer, B. J. \& Lankarani, H. M., Joint clearances with lubricated long bearings in multibody mechanical systems, Journal of Mechanical Design, 122, pp. 484-488, 2000.

[8] ESDU-78035 Tribology Series, Contact Phenomena. I: Stresses, Deflections And Contact Dimensions For Normally-Loaded Unlubricated Elastic Components, Engineering Sciences Data Unit, London, 1978.

[9] Radzimovsky, E.I., Stress Distribution And Strength Conditions Of Two Rolling Cylinders Pressed Together, Eng. Exp. Sta. Univ. Ill., Bull. 408, 1953.

[10] Goldsmith, W., Impact: The Theory and Physical Behaviour of Colliding Solids, Edward Arnold Ltd, London, 1960.

[11] Dubowsky, S. \& Freudenstein, F., Dynamic analysis of mechanical systems with clearances, Part 1: Formulation of dynamic model. Journal of Engineering for Industry, Series B, 93(1), pp. 305-309, 1971.

[12] Pereira, C., Ramalho, A. \& Ambrósio, J., An enhanced cylinder contact force model, submitted to Journal of Mechanical Design, 2011.

[13] Parisch, H., A consistent tangent stiffness matrix for three-dimensional non-linear contact analysis. International Journal for Numerical Methods in Engineering, 28, pp. 1803-1812, 1989.

[14] Knight, M.G., Lacerda, L.A., Wrobel, L.C. \& Henshall, J.L., Parametric study of the contact stresses around spherical and cylindrical inclusions. Computational Materials Science, 25, pp. 115-121, 2002.

[15] Sabelkin, V. \& Mall, S., Relative slip on contact surface under partial slip fretting fatigue condition, Strain, 42, pp. 11-20, 2006.

[16] Zhang, X.C., Xu, B.S., Wang, H.D., Wu, Y.X. \& Jiang, Y., Hertzian contact response of single-layer, functionally graded and sandwich coatings. Materials and Design, 28, pp. 47-54, 2007.

[17] Pei, L., Hyun, S., Molinari, J.F. \& Robbins, M. O., Finite element modeling of elasto-plastic contact between rough surfaces. Journal of the Mechanics and Physics of Solids, 53, pp. 2385-2409, 2005.

[18] Jackson, R. L. \& Green, I., A statistical model of elasto-plastic asperity contact between rough surfaces. Tribology International, 39, pp. 906-914, 2006.

[19] Williams, J.A., The influence of repeated loading, residual stresses and shakedown on the behaviour of tribological contacts. Tribology International, 38, pp. 786-797, 2005.

[20] Masen, M.A., Rooij, M. B. \& Schipper, D.J., Micro-contact based modelling of abrasive wear. Wear, 258, pp. 339-348, 2005.

[21] Wang, L., Xu, J., Yana, L., Liu, Z. \& Yang, G., A FEM study on the mechanical responses of pseudoelastic TiNi alloys to a particle normal loads. Wear, 260, pp. 573-579, 2006.

[22] Marc, MARC User Information, MARC Analysis Research Corporation, Palo Alto, 2000. 